\title{
Some New Half-Linear Integral Inequalities on Time Scales and Applications
}

\author{
Haidong Liu \\ School of Mathematical Sciences, Qufu Normal University, Shandong 273165, China \\ Correspondence should be addressed to Haidong Liu; tomlhd983@163.com
}

Received 13 April 2019; Accepted 23 May 2019; Published 16 June 2019

Academic Editor: Zhengqiu Zhang

Copyright (C) 2019 Haidong Liu. This is an open access article distributed under the Creative Commons Attribution License, which permits unrestricted use, distribution, and reproduction in any medium, provided the original work is properly cited.

The main aim of this paper is to establish some new half-linear integral inequalities on time scales. Our results not only extend and complement some known integral inequalities, but also provide a handy tool for the study of qualitative properties of solutions of some dynamic equations.

\section{Introduction}

The study of dynamic equations on time scales is now an important object of research and has been extensively studied in recent years (see [1-21]). This is due to the fact that the theory of time scales which was introduced by Stefan Hilger [22] can unify and extend the difference and differential calculus in a consistent way.

Because dynamic inequalities play an important role in the study of qualitative properties of solutions of dynamic equations on time scales, many authors have expounded on various classes of dynamic inequalities in recent years; see [23-41] and the references cited therein. For instance, in 2013, Sun and Hassan [32] investigated the nonlinear integral inequality on time scales

$$
\begin{gathered}
y(t) \leq f(t)+g(t) \int_{t_{0}}^{t}\left[a(s) y(s)+b(s) y^{\alpha}(\sigma(s))\right. \\
\left.-c(s) y^{\beta}(\sigma(s))\right] \Delta s,
\end{gathered}
$$

where $y, f, g, a, b, c: \mathbb{T}^{\kappa} \longrightarrow \mathbb{R}_{+}$are rd-continuous functions and $\alpha, \beta$ are positive constants such that $0<\alpha<1<\beta$.

Tian et al. [37] investigated the nonlinear integral inequality on time scales

$$
y^{p}(t) \leq f(t)+g(t) \int_{t_{0}}^{t}\left[a(s) y^{p}(s)+b(s) y^{q}(s)\right.
$$

$$
\begin{aligned}
& +c(s)+\int_{t_{0}}^{s} m(\tau) y^{r}(\tau) \Delta \tau+h_{1}(s) y^{\alpha}(\sigma(s)) \\
& \left.-h_{2}(s) y^{\beta}(\sigma(s))\right] \Delta s, \quad t \in \mathbb{T}^{\kappa},
\end{aligned}
$$

where $p \geq q>0, p \geq r>0,0<\alpha<p<\beta, p, q, r, \alpha$ and $\beta$ are real constants and $y, f, g, a, b, c, m, h_{1}, h_{2}: \mathbb{T}^{\mathcal{K}} \longrightarrow \mathbb{R}_{+}$are rd-continuous functions.

We note that the inequalities (1) and (2) have been proved in the cases when $0<\alpha<1<\beta$ and $0<\alpha<p<\beta$, respectively. So it would be interesting to find the explicit bound for $u$ of (2) in the following two cases when $0<p<$ $\alpha<\beta$ or $0<\beta<\alpha<p$.

In the present paper, we study some new half-linear integral inequalities on time scales. Our results not only complement the results established in [37] in the sense that the results can be applied in cases when $0<p<\alpha<\beta$ or $0<\beta<\alpha<p$, but also furnish a handy tool for the study of qualitative properties of solutions of some complicated dynamic equations.

\section{Preliminaries}

In what follows, we always assume that $\mathbb{R}_{+}=[0, \infty), \mathbb{T}$ is an arbitrary time scale. The following lemmas are useful in the proof of the main results of this paper. 
Lemma 1. Let $m>0, n>0, p>0, \alpha>0$, and $\beta>0$ be given; then for each $x \geq 0$,

$$
m x^{\alpha}-n x^{\beta} \leq \frac{m(\beta-\alpha)}{\beta-p}\left(\frac{(\beta-p) n}{(\alpha-p) m}\right)^{(\alpha-p) /(\alpha-\beta)} x^{p}
$$

holds for the cases when $0<p<\alpha<\beta$ or $0<\beta<\alpha<p$.

Proof. If $x=0$, then it is easy to see that the inequality (3) holds. So we only prove that the inequality (3) holds in the case of $x>0$. For the case $0<p<\alpha<\beta$, set $F(x)=m x^{\alpha-p}-$ $n x^{\beta-p}, x>0$, where $m>0$ and $n>0$. Letting $F^{\prime}(x)=0$, we get $x_{0}=(m(\alpha-p) / n(\beta-p))^{1 /(\beta-\alpha)}$. Since $x \in\left(0, x_{0}\right)$, $F^{\prime}(x)>0 ; x \in\left(x_{0},+\infty\right), F^{\prime}(x)<0, F$ attains its maximum at $x_{0}=(m(\alpha-p) / n(\beta-p))^{1 /(\beta-\alpha)}$ and $F_{\max }=F\left(x_{0}\right)=(m(\beta-$ $\alpha) /(\beta-p))((\beta-p) n /(\alpha-p) m)^{(\alpha-p) /(\alpha-\beta)}$. Thus, (3) holds. For the case $0<\beta<\alpha<p$, by a similar argument with the case $p<\alpha<\beta$, we can get that (3) holds. The proof is complete.

Lemma 2 (see [42]). Assuming that $y \geq 0, p \geq q \geq 0$, and $p \neq 0$, then for any $K>0$,

$$
y^{q / p} \leq \frac{q}{p} K^{(q-p) / p} y+\frac{p-q}{p} K^{q / p} .
$$

Lemma 3 (see [1, Theorem 1.117]). Suppose that for each $\varepsilon>0$ there exists a neighborhood $U$ oft, independent of $\tau \in\left[t_{0}, \sigma(t)\right]$, such that

$$
\begin{aligned}
& \left|w(\sigma(t), \tau)-w(s, \tau)-w_{t}^{\Delta}(t, \tau)(\sigma(t)-s)\right| \\
& \quad \leq \varepsilon|\sigma(t)-s|, \quad s \in U,
\end{aligned}
$$

where $w: \mathbb{T} \times \mathbb{T}^{\mathcal{K}} \longrightarrow \mathbb{R}_{+}$is continuous at $(t, t), t \in \mathbb{T}^{\mathcal{K}}$ with $t>t_{0}$, and $w_{t}^{\Delta}(t, \cdot)$ are rd-continuous on $\left[t_{0}, \sigma(t)\right]$. Then

$$
f(t):=\int_{t_{0}}^{t} w(t, \tau) \Delta \tau
$$

implies

$$
f^{\Delta}(t)=\int_{t_{0}}^{t} w_{t}^{\Delta}(t, \tau) \Delta \tau+w(\sigma(t), t), \quad t \in \mathbb{T}^{\kappa} .
$$

Lemma 4 (see [1, Theorem 6.1]). Suppose that $y$ and $f$ are $r d$-continuous functions and $p \in \mathscr{R}^{+}$. Then

$$
y^{\Delta}(t) \leq p(t) y(t)+f(t), \quad \text { for all } t \in \mathbb{T}
$$

implies

$$
y(t) \leq y\left(t_{0}\right) e_{p}\left(t, t_{0}\right)+\int_{t_{0}}^{t} e_{p}(t, \sigma(\tau)) f(\tau) \Delta \tau
$$

for all $t \in \mathbb{T}$.

\section{Main Results}

In this section, we deal with some half-line inequalities on time scales. For convenience, we always assume that $t \geq t_{0}, t \in$ $\mathbb{T}^{\kappa}$.
Theorem 5. Assume that $x, f, a, b, c, l, m, n: \mathbb{T}^{\kappa} \longrightarrow \mathbb{R}_{+}$, $g, h, k: \mathbb{T}^{\kappa} \longrightarrow(0, \infty)$ are $r d$-continuous functions, $(l / g)^{\Delta}(t) \geq 0, \mu(t) F(t)<1$ for $t \in \mathbb{T}^{\kappa}, \theta: \mathbb{T}^{\kappa} \longrightarrow \mathbb{T}^{\kappa}$ is continuous function satisfying $\theta(t) \leq t$ and $\theta\left(t_{0}\right)=t_{0}$ for $t \epsilon$ $\mathbb{T}^{\kappa}, p, q, r, \alpha$, and $\beta$ are constants satisfying (i) $0 \leq q \leq p, 0 \leq$ $r \leq p, p<\alpha<\beta$ or (ii) $0 \leq q \leq p, 0 \leq r \leq p, 0<\beta<\alpha<p$. Suppose that $x$ satisfies

$$
\begin{gathered}
x^{p}(t) \leq f(t)+g(t) \int_{t_{0}}^{t}\left[a(s) x^{p}(s)+b(s) x^{q}(s)\right. \\
\left.+c(s)+m(s) \int_{\theta\left(t_{0}\right)}^{\theta(s)} n(\xi) x^{r}(\xi) \Delta \xi\right] \Delta s+l(t) \\
\cdot \int_{t_{0}}^{t}\left[h(s) x^{\alpha}(\sigma(s))-k(s) x^{\beta}(\sigma(s))\right] \Delta s,
\end{gathered}
$$

$t \in \mathbb{T}^{\kappa}$,

then

$$
\begin{array}{r}
x(t) \leq\left(f(t)+g(t) \int_{t_{0}}^{t} e_{G \oplus L}(t, \sigma(s)) A(s) \Delta s\right)^{1 / p}, \\
\text { for any } K_{1}>0, K_{2}>0, t \in \mathbb{T}^{\kappa},
\end{array}
$$

where

$$
\begin{gathered}
F(t):=\left(\frac{l}{g}\right)^{\Delta}(t) \int_{t_{0}}^{t} H(s) g(\sigma(s)) \Delta s+\frac{l(\sigma(t))}{g(\sigma(t))} \\
\cdot H(t) g(\sigma(t)),
\end{gathered}
$$

$$
\begin{aligned}
G(t) & :=\frac{F(t)}{1-\mu(t) F(t)}, \\
L(t) & :=a(t) g(t)+\frac{q}{p} K_{1}^{(q-p) / p} b(t) g(t)+\frac{r}{p} \\
\cdot & K_{2}^{(r-p) / p} m(t) \int_{\theta\left(t_{0}\right)}^{\theta(t)} n(\xi) g(\xi) \Delta \xi, \\
M(t) & :=a(t) f(t)+\frac{q}{p} K_{1}^{(q-p) / p} b(t) f(t)+\frac{p-q}{p} \\
\cdot & K_{1}^{q / p} b(t)+c(t)+m(t)
\end{aligned}
$$

$$
\begin{aligned}
& \cdot \int_{\theta\left(t_{0}\right)}^{\theta(t)} n(\xi)\left[\frac{r}{p} K_{2}^{(r-p) / p} f(\xi)+\frac{p-r}{p} K_{2}^{r / p}\right] \Delta \xi \\
& +\left(\frac{l}{g}\right)^{\Delta}(t) \int_{t_{0}}^{t} H(s) f(\sigma(s)) \Delta s+\frac{l(\sigma(t))}{g(\sigma(t))} H(t) \\
& \cdot f(\sigma(t)),
\end{aligned}
$$




$$
\begin{aligned}
& H(t):=\frac{h(t)(\beta-\alpha)}{\beta-p}\left(\frac{(\beta-p) k(t)}{(\alpha-p) h(t)}\right)^{(\alpha-p) /(\alpha-\beta)}, \\
& A(t):=(1+\mu(t) G(t)) M(t) .
\end{aligned}
$$

Proof. From Lemma 1 and (10), we get that

$$
\begin{aligned}
& x^{p}(t) \leq f(t)+g(t) \int_{t_{0}}^{t}\left[a(s) x^{p}(s)+b(s) x^{q}(s)\right. \\
& \left.+c(s)+m(s) \int_{\theta\left(t_{0}\right)}^{\theta(s)} n(\xi) x^{r}(\xi) \Delta \xi\right] \Delta s+l(t) \\
& \cdot \int_{t_{0}}^{t}\left[h(s) x^{\alpha}(\sigma(s))-k(s) x^{\beta}(\sigma(s))\right] \Delta s \\
& \leq f(t)+g(t) \int_{t_{0}}^{t}\left[a(s) x^{p}(s)+b(s) x^{q}(s)+c(s)\right. \\
& \left.+m(s) \int_{\theta\left(t_{0}\right)}^{\theta(s)} n(\xi) x^{r}(\xi) \Delta \xi\right] \Delta s+l(t) \\
& \cdot \int_{t_{0}}^{t} H(s) x^{p}(\sigma(s)) \Delta s, \quad t \in \mathbb{T}^{\kappa},
\end{aligned}
$$

where $H(t)$ is defined as in (16). Denote

$$
\begin{gathered}
z(t)=\int_{t_{0}}^{t}\left[a(s) x^{p}(s)+b(s) x^{q}(s)+c(s)\right. \\
\left.+m(s) \int_{\theta\left(t_{0}\right)}^{\theta(s)} n(\xi) x^{r}(\xi) \Delta \xi\right] \Delta s+\frac{l(t)}{g(t)} \\
\cdot \int_{t_{0}}^{t} H(s) x^{p}(\sigma(s)) \Delta s, \quad t \in \mathbb{T}^{\kappa} .
\end{gathered}
$$

From the assumptions on $x, a, b, c, m, n, \theta, g, l, H,(18)$ and (19), we obtain that $z$ is nondecreasing and

$$
x(t) \leq(f(t)+g(t) z(t))^{1 / p}, \quad t \in \mathbb{T}^{\kappa} .
$$

Combining (19) and (20), we have that

$$
\begin{gathered}
z^{\Delta}(t)=a(t) x^{p}(t)+b(t) x^{q}(t)+c(t)+m(t) \\
\cdot \int_{\theta\left(t_{0}\right)}^{\theta(t)} n(\xi) x^{r}(\xi) \Delta \xi+\left(\frac{l}{g}\right)^{\Delta}(t)
\end{gathered}
$$

$$
\begin{aligned}
& \cdot \int_{t_{0}}^{t} H(s) x^{p}(\sigma(s)) \Delta s+\frac{l(\sigma(t))}{g(\sigma(t))} H(t) x^{p}(\sigma(t)) \\
& \leq a(t)(f(t)+g(t) z(t))+b(t) \\
& \cdot(f(t)+g(t) z(t))^{q / p}+c(t)+m(t) \\
& \cdot \int_{\theta\left(t_{0}\right)}^{\theta(t)} n(\xi)(f(\xi)+g(\xi) z(\xi))^{r / p} \Delta \xi+\left(\frac{l}{g}\right)^{\Delta}(t) \\
& \cdot \int_{t_{0}}^{t} H(s)(f(\sigma(s))+g(\sigma(s)) z(\sigma(s))) \Delta s \\
& +\frac{l(\sigma(t))}{g(\sigma(t))} H(t)(f(\sigma(t))+g(\sigma(t)) z(\sigma(t))), \\
& \quad t \in \mathbb{T}^{\kappa} .
\end{aligned}
$$

Applying Lemma 2 on the right side of (21), we get that

$$
\begin{aligned}
& z^{\Delta}(t) \leq a(t)(f(t)+g(t) z(t))+b(t)\left[\frac{q}{p}\right. \\
& \left.\cdot K_{1}^{(q-p) / p}(f(t)+g(t) z(t))+\frac{p-q}{p} K_{1}^{q / p}\right]+c(t) \\
& +m(t) \int_{\theta\left(t_{0}\right)}^{\theta(t)} n(\xi)\left[\frac{r}{p} K_{2}^{(r-p) / p}(f(\xi)+g(\xi) z(\xi))\right. \\
& \left.+\frac{p-r}{p} K_{2}^{r / p}\right] \Delta \xi+\left(\frac{l}{g}\right)^{\Delta}(t) \int_{t_{0}}^{t} H(s) \\
& \cdot(f(\sigma(s))+g(\sigma(s)) z(\sigma(s))) \Delta s \\
& +\frac{l(\sigma(t))}{g(\sigma(t))} H(t)(f(\sigma(t))+g(\sigma(t)) z(\sigma(t))) \\
& \leq a(t) f(t)+a(t) g(t) z(t)+\frac{q}{p} K_{1}^{(q-p) / p} b(t) \\
& \cdot(f(t)+g(t) z(t))+\frac{p-q}{p} K_{1}^{q / p} b(t)+c(t)+\frac{r}{p}
\end{aligned}
$$

$\cdot K_{2}^{(r-p) / p} z(t) m(t) \int_{\theta\left(t_{0}\right)}^{\theta(t)} n(\xi) g(\xi) \Delta \xi+m(t)$

$\cdot \int_{\theta\left(t_{0}\right)}^{\theta(t)} n(\xi)\left[\frac{r}{p} K_{2}^{(r-p) / p} f(\xi)+\frac{p-r}{p} K_{2}^{r / p}\right] \Delta \xi$

$+z(\sigma(t))\left(\frac{l}{g}\right)^{\Delta}(t) \int_{t_{0}}^{t} H(s) g(\sigma(s)) \Delta s+\left(\frac{l}{g}\right)^{\Delta}$

$\cdot(t) \int_{t_{0}}^{t} H(s) f(\sigma(s)) \Delta s+z(\sigma(t)) \frac{l(\sigma(t))}{g(\sigma(t))} H(t)$

$\cdot g(\sigma(t))+\frac{l(\sigma(t))}{g(\sigma(t))} H(t) f(\sigma(t))=L(t) z(t)$

$+F(t) z(\sigma(t))+M(t)$, 
where $F(t), L(t)$, and $M(t)$ are defined as in (12), (14), and (15). From (13), we get

$$
F(t)=\frac{G(t)}{1+\mu(t) G(t)},
$$

and by (22), we obtain

$$
\begin{aligned}
z^{\Delta}(t) \leq & L(t) z(t)+\frac{G(t)}{1+\mu(t) G(t)} z(\sigma(t))+M(t) \\
= & L(t) z(t) \\
& +\frac{G(t)}{1+\mu(t) G(t)}\left[z(t)+\mu(t) z^{\Delta}(t)\right] \\
& +M(t), \quad t \in \mathbb{T}^{\kappa} .
\end{aligned}
$$

Hence

$$
\begin{aligned}
\frac{1}{1+\mu(t) G(t)} z^{\Delta}(t) \leq & {\left[\frac{G(t)}{1+\mu(t) G(t)}+L(t)\right] z(t) } \\
& +M(t), \quad t \in \mathbb{T}^{\kappa},
\end{aligned}
$$

equivalently

$$
\begin{aligned}
z^{\Delta}(t) \leq & {[G(t)+(1+\mu(t) G(t)) L(t)] z(t) } \\
& +(1+\mu(t) G(t)) M(t) \\
= & (G \oplus L)(t) z(t)+(1+\mu(t) G(t)) M(t) \\
= & (G \oplus L)(t) z(t)+A(t), \quad t \in \mathbb{T}^{\kappa},
\end{aligned}
$$

where $A(t)$ is defined as in (17). Note that $z$ is rd-continuous and $G \oplus L \in \mathscr{R}^{+}$; from Lemma 4 and (26), we have that

$$
z(t) \leq \int_{t_{0}}^{t} e_{G \oplus L}(t, \sigma(s)) A(s) \Delta s, \quad t \in \mathbb{T}^{\kappa} .
$$

This and (20) imply the desired inequality (11). This completes the proof.

If we let $g(t)=l(t), m(t)=1$, and $\theta(t)=t$ in Theorem 5, then we obtain the following corollary.

Corollary 6. Assume that $x, a, b, c, f, g, n, h, k, p, q, r, \alpha, \beta$ and $H$ are defined the same as in Theorem 5 and $\mu(t) F(t)<1$ for $t \in \mathbb{T}^{\kappa}$. Suppose that $u$ satisfies

$$
\begin{gathered}
x^{p}(t) \leq f(t)+g(t) \int_{t_{0}}^{t}\left[a(s) x^{p}(s)+b(s) x^{q}(s)\right. \\
+c(s)+\int_{t_{0}}^{s} n(\xi) x^{r}(\xi) \Delta \xi+h(s) x^{\alpha}(\sigma(s)) \\
\left.\quad-k(s) x^{\beta}(\sigma(s))\right] \Delta s, \quad t \in \mathbb{T}^{\kappa},
\end{gathered}
$$

then

$$
x(t) \leq\left(f(t)+g(t) \int_{t_{0}}^{t} e_{G \oplus L}(t, \sigma(s)) A(s) \Delta s\right)^{1 / p},
$$

$$
\text { for any } K_{1}>0, K_{2}>0, t \in \mathbb{T}^{\kappa} \text {, }
$$

where

$$
\begin{aligned}
F(t) & :=H(t) g(\sigma(t)), \\
G(t) & :=\frac{F(t)}{1-\mu(t) F(t)}, \\
L(t) & :=a(t) g(t)+\frac{q}{p} K_{1}^{(q-p) / p} b(t) g(t) \\
& +\frac{r}{p} K_{2}^{(r-p) / p} \int_{t_{0}}^{t} n(\xi) g(\xi) \Delta \xi,
\end{aligned}
$$

$M(t)$

$$
\begin{aligned}
:= & a(t) f(t)+\frac{q}{p} K_{1}^{(q-p) / p} b(t) f(t) \\
& +\frac{p-q}{p} K_{1}^{q / p} b(t)+c(t) \\
& +\int_{t_{0}}^{t} n(\xi)\left[\frac{r}{p} K_{2}^{(r-p) / p} f(\xi)+\frac{p-r}{p} K_{2}^{r / p}\right] \Delta \xi \\
& +H(t) f(\sigma(t)), \\
A(t): & =(1+\mu(t) G(t)) M(t) .
\end{aligned}
$$

Remark 7. Corollary 6 extends Theorem 2.1 in [37] to the cases $p<\alpha<\beta$ and $0<\beta<\alpha<p$.

Remark 8. If $l(t)=0, c(t)=0$, and $m(t)=0$, then Theorem 5 reduces to Theorem 3.2 in [28]. If $l(t)=0, a(t)=0$, and $m(t)=0$, then Theorem 5 reduces to Theorem 2.4 in [29].

Theorem 9. Assume that $x, a, b, c, f, g, l, m, n, \theta, h, k, p, q$, $r, \alpha, \beta, H$, and $L$ are defined the same as in Theorem 5 , and $\mu(t) \widetilde{F}(t)<1$ for $t \in \mathbb{T}^{\kappa}$. Let $w(t, s)$ and $v(t, s)$ be defined as in Lemma 3 such that $w_{t}^{\Delta}(t, s) \geq 0$ and $v_{t}^{\Delta}(t, s) \geq 0$ for $t \geq s$ and (5) holds. Suppose that $x$ satisfies

$$
\begin{aligned}
& x^{p}(t) \leq f(t)+g(t) \int_{t_{0}}^{t} w(t, s)\left[a(s) x^{p}(s)\right. \\
& +b(s) x^{q}(s)+c(s) \\
& \left.+m(s) \int_{\theta\left(t_{0}\right)}^{\theta(s)} n(\xi) x^{r}(\xi) \Delta \xi\right] \Delta s+l(t) \\
& \cdot \int_{t_{0}}^{t} v(t, s)\left[h(s) x^{\alpha}(\sigma(s))-k(s) x^{\beta}(\sigma(s))\right] \Delta s,
\end{aligned}
$$$$
t \in \mathbb{T}^{\kappa}
$$

then

$$
x(t) \leq\left(f(t)+g(t) \int_{t_{0}}^{t} e_{\widetilde{G} \oplus \widetilde{L}}(t, \sigma(s)) \widetilde{A}(s) \Delta s\right)^{1 / p},
$$


where

$$
\begin{aligned}
& \widetilde{F}(t):=\left(\frac{l}{g}\right)^{\Delta}(t) \int_{t_{0}}^{t} v(t, s) H(s) g(\sigma(s)) \Delta s \\
& +\frac{l(\sigma(t))}{g(\sigma(t))}\left[\int_{t_{0}}^{t} v_{t}^{\Delta}(t, s) H(s) g(\sigma(s)) \Delta s\right. \\
& +v(\sigma(t), t) H(t) g(\sigma(t))], \\
& \widetilde{G}(t):=\frac{\widetilde{F}(t)}{1-\mu(t) \widetilde{F}(t)}, \\
& \widetilde{L}(t):=\int_{t_{0}}^{t} w_{t}^{\Delta}(t, s) L(s) \Delta s+w(\sigma(t), t) L(t), \\
& \widetilde{M}(t):=\int_{t_{0}}^{t} w_{t}^{\Delta}(t, s)\left\{a(s) f(s)+b(s)\left[\frac{q}{p}\right.\right. \\
& \left.\cdot K_{1}^{(q-p) / p} f(s)+\frac{p-q}{p} K_{1}^{q / p}\right]+c(s)+m(s) \\
& \cdot \int_{\theta\left(t_{0}\right)}^{\theta(s)} n(\xi) \\
& \left.\cdot\left[\frac{r}{p} K_{2}^{(r-p) / p} f(\xi)+\frac{p-r}{p} K_{2}^{r / p}\right] \Delta \xi\right\} \Delta s \\
& +w(\sigma(t), t)\left\{a(t) f(t)+b(t)\left[\frac{q}{p} K_{1}^{(q-p) / p} f(t)\right.\right. \\
& \left.+\frac{p-q}{p} K_{1}^{q / p}\right]+c(t)+m(t) \int_{\theta\left(t_{0}\right)}^{\theta(t)} n(\xi)\left[\frac{r}{p}\right. \\
& \left.\left.\cdot K_{2}^{(r-p) / p} f(\xi)+\frac{p-r}{p} K_{2}^{r / p}\right] \Delta \xi\right\}+\left(\frac{l}{g}\right)^{\Delta} \\
& \cdot(t) \int_{t_{0}}^{t} v(t, s) H(s) f(\sigma(s)) \Delta s \\
& +\frac{l(\sigma(t))}{g(\sigma(t))}\left[\int_{t_{0}}^{t} v_{t}^{\Delta}(t, s) H(s) f(\sigma(s)) \Delta s+v(\sigma(t), t)\right. \\
& \cdot H(t) f(\sigma(t))] \text {, } \\
& \widetilde{A}(t):=(1+\mu(t) \widetilde{G}(t)) \widetilde{M}(t),
\end{aligned}
$$

and $L(t)$ is defined as in (14).

Proof. From Lemma 1 and (34), we get that

$$
\begin{aligned}
& x^{p}(t) \leq f(t)+g(t) \int_{t_{0}}^{t} w(t, s)\left[a(s) x^{p}(s)\right. \\
& \quad+b(s) x^{q}(s)+c(s) \\
& \left.\quad+m(s) \int_{\theta\left(t_{0}\right)}^{\theta(s)} n(\xi) x^{r}(\xi) \Delta \xi\right] \Delta s+l(t)
\end{aligned}
$$

$$
\begin{aligned}
& \cdot \int_{t_{0}}^{t} v(t, s)\left[h(s) x^{\alpha}(\sigma(s))-k(s) x^{\beta}(\sigma(s))\right] \Delta s \\
& \leq f(t)+g(t) \int_{t_{0}}^{t} w(t, s)\left[a(s) x^{p}(s)+b(s) x^{q}(s)\right. \\
& \left.+c(s)+m(s) \int_{\theta\left(t_{0}\right)}^{\theta(s)} n(\xi) x^{r}(\xi) \Delta \xi\right] \Delta s+l(t) \\
& \cdot \int_{t_{0}}^{t} v(t, s) H(s) x^{p}(\sigma(s)) \Delta s, \quad t \in \mathbb{T}^{\kappa},
\end{aligned}
$$

where $H(t)$ is defined as in (16). Denote

$$
\begin{gathered}
z(t)=\int_{t_{0}}^{t} w(t, s)\left[a(s) x^{p}(s)+b(s) x^{q}(s)+c(s)\right. \\
\left.+m(s) \int_{\theta\left(t_{0}\right)}^{\theta(s)} n(\xi) x^{r}(\xi) \Delta \xi\right] \Delta s+\frac{l(t)}{g(t)} \\
\cdot \int_{t_{0}}^{t} v(t, s) H(s) x^{p}(\sigma(s)) \Delta s, \quad t \in \mathbb{T}^{\kappa} .
\end{gathered}
$$

From the assumptions on $x, w, v, a, b, c, m, n, \theta, g, l, H,(42)$ and (43), we obtain that $z$ is nondecreasing and

$$
x(t) \leq(f(t)+g(t) z(t))^{1 / p}, \quad t \in \mathbb{T}^{\kappa} .
$$

Combining (43) and (44), we have that

$$
\begin{gathered}
z^{\Delta}(t)=\int_{t_{0}}^{t} w_{t}^{\Delta}(t, s)\left[a(s) x^{p}(s)+b(s) x^{q}(s)+c(s)\right. \\
\left.+m(s) \int_{\theta\left(t_{0}\right)}^{\theta(s)} n(\xi) x^{r}(\xi) \Delta \xi\right] \Delta s+w(\sigma(t), t) \\
\cdot\left[a(t) x^{p}(t)+b(t) x^{q}(t)+c(t)+m(t)\right. \\
\left.\cdot \int_{\theta\left(t_{0}\right)}^{\theta(t)} n(\xi) x^{r}(\xi) \Delta \xi\right]+\left(\frac{l}{g}\right)^{\Delta}(t) \int_{t_{0}}^{t} v(t, s) \\
\cdot H(s) x^{p}(\sigma(s)) \Delta s+\frac{l(\sigma(t))}{g(\sigma(t))}\left[\int_{t_{0}}^{t} v_{t}^{\Delta}(t, s)\right. \\
\left.\cdot H(s) x^{p}(\sigma(s)) \Delta s+v(\sigma(t), t) H(t) x^{p}(\sigma(t))\right] \\
\leq \int_{t_{0}}^{t} w_{t}^{\Delta}(t, s)[a(s)(f(s)+g(s) z(s))+b(s) \\
\cdot(f(s)+g(s) z(s))^{q / p}+c(s)+m(s)
\end{gathered}
$$




$$
\begin{aligned}
& \left.\cdot \int_{\theta\left(t_{0}\right)}^{\theta(s)} n(\xi)(f(\xi)+g(\xi) z(\xi))^{r / p} \Delta \xi\right] \Delta s \\
& +w(\sigma(t), t)[a(t)(f(t)+g(t) z(t))+b(t)(f(t) \\
& +g(t) z(t))^{q / p}+c(t)+m(t) \int_{\theta\left(t_{0}\right)}^{\theta(t)} n(\xi) \\
& \cdot(f(\xi)+g(\xi) z(\xi))^{r / p} \Delta \xi+\left(\frac{l}{g}\right)^{\Delta}(t) \\
& \cdot \int_{t_{0}}^{t} v(t, s) H(s) \\
& \cdot(f(\sigma(s))+g(\sigma(s)) z(\sigma(s))) \Delta s \\
& +\frac{l(\sigma(t))}{g(\sigma(t))}\left[\int_{t_{0}}^{t} v_{t}^{\Delta}(t, s) H(s)\right. \\
& \cdot(f(\sigma(s))+g(\sigma(s)) z(\sigma(s))) \Delta s+v(\sigma(t), t) \\
& \cdot H(t)(f(\sigma(t))+g(\sigma(t)) z(\sigma(t)))], t \in \mathbb{T}^{\kappa} .
\end{aligned}
$$

Applying Lemma 2 on the right side of (45), we have that

$$
\begin{aligned}
& z^{\Delta}(t) \leq \int_{t_{0}}^{t} w_{t}^{\Delta}(t, s)\{a(s)(f(s)+g(s) z(s))+b(s) \\
& \cdot\left[\frac{q}{p} K_{1}^{(q-p) / p}(f(s)+g(s) z(s))+\frac{p-q}{p} K_{1}^{q / p}\right] \\
& +c(s)+m(s) \int_{\theta\left(t_{0}\right)}^{\theta(s)} n(\xi) \\
& \cdot\left[\frac{r}{p} K_{2}^{(r-p) / p}(f(\xi)+g(\xi) z(\xi))\right. \\
& \left.\left.+\frac{p-r}{p} K_{2}^{r / p}\right] \Delta \xi\right\} \Delta s+w(\sigma(t), t)\{a(t) \\
& \cdot(f(t)+g(t) z(t))+b(t)\left[\frac{q}{p} K_{1}^{(q-p) / p}(f(t)\right. \\
& \left.+g(t) z(t))+\frac{p-q}{p} K_{1}^{q / p}\right]+c(t)+m(t) \\
& \cdot \int_{\theta\left(t_{0}\right)}^{\theta(t)} n(\xi)\left[\frac{r}{p} K_{2}^{(r-p) / p}(f(\xi)+g(\xi) z(\xi))\right. \\
& \left.\left.+\frac{p-r}{p} K_{2}^{r / p}\right] \Delta \xi\right\}+\left(\frac{l}{g}\right)^{\Delta}(t) \int_{t_{0}}^{t} v(t, s) H(s) \\
& \cdot(f(\sigma(s))+g(\sigma(s)) z(\sigma(s))) \Delta s \\
& +\frac{l(\sigma(t))}{g(\sigma(t))}\left[\int_{t_{0}}^{t} v_{t}^{\Delta}(t, s) H(s)(f(\sigma(s))+g(\sigma(s))\right. \\
& \text { - } z(\sigma(s))) \Delta s+v(\sigma(t), t) H(t)(f(\sigma(t))
\end{aligned}
$$

$$
\begin{aligned}
& +g(\sigma(t)) z(\sigma(t)))] \leq z(t) \int_{t_{0}}^{t} w_{t}^{\Delta}(t, s)[a(s) g(s) \\
& +b(s) \frac{q}{p} K_{1}^{(q-p) / p} g(s)+m(s) \int_{\theta\left(t_{0}\right)}^{\theta(s)} n(\xi) \\
& \left.\cdot \frac{r}{p} K_{2}^{(r-p) / p} g(\xi) \Delta \xi\right] \Delta s+\int_{t_{0}}^{t} w_{t}^{\Delta}(t, s) \\
& \cdot\left\{a(s) f(s)+b(s)\left[\frac{q}{p} K_{1}^{(q-p) / p} f(s)+\frac{p-q}{p}\right.\right. \\
& \left.\cdot K_{1}^{q / p}\right]+c(s)+m(s) \int_{\theta\left(t_{0}\right)}^{\theta(s)} n(\xi)\left[\frac{r}{p} K_{2}^{(r-p) / p} f(\xi)\right. \\
& \left.\left.+\frac{p-r}{p} K_{2}^{r / p}\right] \Delta \xi\right\} \Delta s+z(t) w(\sigma(t), t) \\
& \cdot\left\{\begin{array}{l}
\theta(t) \\
a(t) g(t)+b(t)\left[\frac{q}{p} K_{1}^{(q-p) / p} g(t)\right]+m(t)
\end{array}\right. \\
& +\theta\left(t_{0}\right) \\
& \left.\int_{2}^{(r-p) / p} g(\xi) \Delta \xi\right\}+w(\sigma(t), t)
\end{aligned}
$$$$
\left\{a(t) f(t)+b(t)\left[\frac{q}{p} K_{1}^{(q-p) / p} f(t)+\frac{p-q}{p}\right.\right.
$$$$
\left.\cdot K_{1}^{q / p}\right]+c(t)+m(t) \int_{\theta\left(t_{0}\right)}^{\theta(t)} n(\xi)\left[\frac{r}{p}\right.
$$$$
\left.\left.\cdot K_{2}^{q / p(r-p) / p} f(\xi)+\frac{p-r}{p} K_{2}^{r / p}\right] \Delta \xi\right\}
$$$$
+z(\sigma(t))\left\{\left(\frac{l}{g}\right)^{\Delta}(t) \int_{t_{0}}^{t} v(t, s) H(s) g(\sigma(s)) \Delta s\right.
$$$$
+\frac{l(\sigma(t))}{g(\sigma(t))}\left[\int_{t_{0}}^{t} v_{t}^{\Delta}(t, s) H(s) g(\sigma(s)) \Delta s\right.
$$$$
+v(\sigma(t), t) H(t) g(\sigma(t))]\}+\left(\frac{l}{g}\right)^{\Delta}(t)
$$$$
\cdot \int_{t_{0}}^{t} v(t, s) H(s) f(\sigma(s)) \Delta s
$$$$
+\frac{l(\sigma(t))}{g(\sigma(t))}\left[\int_{t_{0}}^{t} v_{t}^{\Delta}(t, s) H(s) f(\sigma(s)) \Delta s\right.
$$$$
+v(\sigma(t), t) H(t) f(\sigma(t))] \leq\left(\int_{t_{0}}^{t} w_{t}^{\Delta}(t, s)\right.
$$$$
\cdot L(s) \Delta s+w(\sigma(t), t) L(t)) z(t)+\left\{\left(\frac{l}{g}\right)^{\Delta}\right.
$$$$
\cdot(t) \int_{t_{0}}^{t} v(t, s) H(s) g(\sigma(s)) \Delta s
$$$$
+\frac{l(\sigma(t))}{g(\sigma(t))}\left[\int_{t_{0}}^{t} v_{t}^{\Delta}(t, s) H(s) g(\sigma(s)) \Delta s\right.
$$ 


$$
\begin{aligned}
& +v(\sigma(t), t) H(t) g(\sigma(t))]\} z(\sigma(t))+\int_{t_{0}}^{t} w_{t}^{\Delta}(t, \\
& s)\left\{a(s) f(s)+b(s)\left[\frac{q}{p} K_{1}^{(q-p) / p} f(s)+\frac{p-q}{p}\right.\right. \\
& \left.\cdot K_{1}^{q / p}\right]+c(s)+m(s) \int_{\theta\left(t_{0}\right)}^{\theta(s)} n(\xi)\left[\frac{r}{p} K_{2}^{(r-p) / p} f(\xi)\right. \\
& \left.\left.+\frac{p-r}{p} K_{2}^{r / p}\right] \Delta \xi\right\} \Delta s+w(\sigma(t), t)\{a(t) \\
& \cdot f(t)+b(t)\left[\frac{q}{p} K_{1}^{(q-p) / p} f(t)+\frac{p-q}{p} K_{1}^{q / p}\right] \\
& +c(t)+m(t) \int_{\theta\left(t_{0}\right)}^{\theta(t)} n(\xi)\left[\frac{r}{p} K_{2}^{(r-p) / p} f(\xi)+\frac{p-r}{p}\right. \\
& \left.\left.\cdot K_{2}^{r / p}\right] \Delta \xi\right\}+\left(\frac{l}{g}\right)^{\Delta}(t) \int_{t_{0}}^{t} v(t, s) H(s) \\
& \cdot f(\sigma(s)) \Delta s+v(\sigma(t), t) H(t) f(\sigma(t))] \\
& =\widetilde{L}(t) z(t)+\widetilde{F}(t) z(\sigma(t))+\widetilde{M}(t), \\
& f(\sigma)) \Delta s+\frac{l(\sigma))}{g(\sigma(t))}\left[\int_{t_{0}}^{t} v_{t}^{\Delta}(t, s) H(s)\right.
\end{aligned}
$$

where $\widetilde{F}(t), \widetilde{L}(t)$, and $\widetilde{M}(t)$ are defined as in (36), (38), and (40). By a similar argument with Theorem 5 in the remaining proof of theorem, one can prove (35). This completes the proof.

If we let $g(t)=l(t), m(t)=1, w(t, s)=v(t, s)$, and $\theta(t)=t$ in Theorem 9 , then we obtain the following corollary.

Corollary 10. Assume that $x, f, g, w, a, b, c, n, h, k, p, q, r, \alpha$, $\beta$, and $H$ are defined the same as in Theorem 9 and $\mu(t) \widetilde{F}(t)<$ 1 for $t \in \mathbb{T}^{\mathcal{K}}$. Suppose that $x$ satisfies

$$
\begin{gathered}
x^{p}(t) \leq f(t)+g(t) \int_{t_{0}}^{t} w(t, s)\left[a(s) x^{p}(s)\right. \\
+b(s) x^{q}(s)+c(s)+\int_{t_{0}}^{s} n(\xi) x^{r}(\xi) \Delta \xi \\
\left.+h(s) x^{\alpha}(\sigma(s))-k(s) x^{\beta}(\sigma(s))\right] \Delta s,
\end{gathered}
$$

$t \in \mathbb{T}^{\kappa}$

then

$$
x(t) \leq\left(f(t)+g(t) \int_{t_{0}}^{t} e_{\widetilde{G} \oplus \widetilde{L}}(t, \sigma(s)) \widetilde{A}(s) \Delta s\right)^{1 / p},
$$

where

$$
\begin{aligned}
& \widetilde{F}(t):=\int_{t_{0}}^{t} w_{t}^{\Delta}(t, s) F(s) \Delta s+w(\sigma(t), t) F(t), \\
& \widetilde{G}(t):=\frac{\widetilde{F}(t)}{1-\mu(t) \widetilde{F}(t)}, \\
& \widetilde{L}(t):=\int_{t_{0}}^{t} w_{t}^{\Delta}(t, s) L(s) \Delta s+w(\sigma(t), t) L(t), \\
& \widetilde{M}(t):=\int_{t_{0}}^{t} w_{t}^{\Delta}(t, s) M(s) \Delta s+w(\sigma(t), t) M(t), \\
& \widetilde{A}(t)=(1+\mu(t) \widetilde{G}(t)) \widetilde{M}(t),
\end{aligned}
$$

and $F(t), L(t)$, and $M(t)$ are defined the same as in (30), (31), and (32).

Remark 11. Corollary 10 extends Theorem 2.2 in [37] to the cases $p<\alpha<\beta$ and $0<\beta<\alpha<p$.

Remark 12. If $c(t)=0, m(t)=0$, and $l(t)=0$, then Theorem 9 reduces to Theorem 3.8 in [28]. If $m(t)=0$ and $l(t)=0$, then Theorem 9 reduces to Theorem 2.11 in [29].

\section{Applications}

In this section, we apply our results to study the boundedness of the solutions of a dynamic equation on time scales.

Example 13. Consider the following dynamic equation on time scales:

$$
\begin{aligned}
& \left(x^{p}(t)\right)^{\Delta} \\
& \quad=P\left(t, x(t), x(\sigma(t)), \int_{\theta\left(t_{0}\right)}^{\theta(t)} Q(s, x(s)) \Delta s\right), \\
& x\left(t_{0}\right)=x_{0}, \quad t_{0}, t \in \mathbb{T}^{\kappa},
\end{aligned}
$$

where $p>0$ and $u_{0}$ are constants, $\theta: \mathbb{T}^{\mathcal{K}} \longrightarrow \mathbb{T}^{\mathcal{K}}$ is continuous function satisfying $\theta(t) \leq t$ and $\theta\left(t_{0}\right)=t_{0}$ for $t \in \mathbb{T}^{\kappa}$, and $P: \mathbb{T}^{\kappa} \times \mathbb{R} \times \mathbb{R} \times \mathbb{R} \longrightarrow \mathbb{R}$ and $Q: \mathbb{T}^{\kappa} \times \mathbb{R} \longrightarrow \mathbb{R}$ are continuous functions.

Theorem 14. Suppose that the functions $P$ and $Q$ in (50) satisfy the conditions

$$
\begin{aligned}
|P(t, u, v, w)| \leq & c(t)+a(t)|u|^{p}+b(t)|u|^{q} \\
& +h(t)|v|^{\alpha}-k(t)|v|^{\beta} \\
& +m(t)|w|
\end{aligned}
$$

$t \in \mathbb{T}^{\kappa}, u, v, w \in \mathbb{R}$,

$$
|Q(t, u)| \leq n(t)|u|^{r}, \quad t \in \mathbb{T}^{\kappa}, u \in \mathbb{R}
$$

where $a, b, c, m, n: \mathbb{T}^{\kappa} \longrightarrow \mathbb{R}_{+}$, and $h, k: \mathbb{T}^{\kappa} \longrightarrow(0, \infty)$ are $r d$-continuous functions and $q, r, \alpha$, and $\beta$ are constants 
satisfying (i) $0 \leq q \leq p, 0 \leq r \leq p, p<\alpha<\beta$ or (ii) $0 \leq q \leq p, 0 \leq r \leq p, 0<\beta<\alpha<p$.

If $x$ is a solution of (50) and $\mu(t) F(t)<1$ for $t \in \mathbb{T}^{\mathcal{K}}$, then

$$
x(t) \leq\left(\left|x_{0}\right|^{p}+\int_{t_{0}}^{t} e_{G \oplus L}(t, \sigma(s)) A(s) \Delta s\right)^{1 / p}
$$

for any $K_{1}>0, K_{2}>0, t \in \mathbb{T}^{\kappa}$,

where

$$
\begin{aligned}
& F(t):=\frac{h(t)(\beta-\alpha)}{\beta-p}\left(\frac{(\beta-p) k(t)}{(\alpha-p) h(t)}\right)^{(\alpha-p) /(\alpha-\beta)}, \\
& G(t):=\frac{F(t)}{1-\mu(t) F(t)}, \\
& L(t):=a(t)+\frac{q}{p} K_{1}^{(q-p) / p} b(t)+\frac{r}{p} K_{2}^{(r-p) / p} m(t) \\
& \cdot \int_{\theta\left(t_{0}\right)}^{\theta(t)} n(\xi) \Delta \xi, \\
& M(t):=\left|x_{0}\right|^{p} a(t)+\frac{q}{p} K_{1}^{(q-p) / p}\left|x_{0}\right|^{p} b(t)+\frac{p-q}{p} \\
& \cdot K_{1}^{q / p} b(t)+c(t) \\
& \quad+\left(\frac{r}{p} K_{2}^{(r-p) / p}\left|x_{0}\right|^{p}+\frac{p-r}{p} K_{2}^{r / p}\right) m(t) \\
& \quad \cdot \int_{\theta\left(t_{0}\right)}^{\theta(t)} n(\xi) \Delta \xi+\left|x_{0}\right|^{p} F(t), \\
& A(t):=(1+\mu(t) G(t)) M(t) .
\end{aligned}
$$

Proof. The equivalent integral equation of (50) is denoted by

$$
\begin{aligned}
& x^{p}(t)=x_{0}^{p}+\int_{t_{0}}^{t} P \\
& \quad\left(s, x(s), x(\sigma(s)), \int_{\theta\left(t_{0}\right)}^{\theta(s)} Q(\xi, x(\xi)) \Delta \xi\right) \Delta s
\end{aligned}
$$

$t \in \mathbb{T}^{\kappa}$.

Using the assumptions (52) and (53), we have

$$
\begin{aligned}
& \left|x^{p}(t)\right| \leq\left|x_{0}\right|^{p}+\int_{t_{0}}^{t}\left[a(s)|x(s)|^{p}+b(s)|x(s)|^{q}\right. \\
& \quad+c(s)
\end{aligned}
$$

$$
\begin{aligned}
& +m(s) \int_{\theta\left(t_{0}\right)}^{\theta(s)} n(\xi)|x(\xi)|^{r} \Delta \xi+h(s)|x(\sigma(s))|^{\alpha} \\
& \left.-k(s)|x(\sigma(s))|^{\beta}\right] \Delta s, \quad t \in \mathbb{T}^{\kappa} .
\end{aligned}
$$

Then, a suitable application of Theorem 5 to (61) yields (54).

\section{Data Availability}

The data used to support the findings of this study are available from the corresponding author upon request.

\section{Conflicts of Interest}

The author declares that there are no conflicts of interest regarding the publication of this article.

\section{Acknowledgments}

The author is indebted to the anonymous referees for their valuable suggestions and helpful comments which helped improve the paper significantly. This research was supported by the Natural Science Foundation of Shandong Province (China) (No. ZR2018MA018) and the National Natural Science Foundation of China (Nos. 11671227 and 61873144).

\section{References}

[1] M. Bohner and A. Peterson, Dynamic Equations on Time Scales: An Introduction with Applications, Birkhäuser, Boston, Mass, USA, 2001.

[2] M. Bohner and A. Peterson, Advances in Dynamic Equations on Time Scales, Birkhäuser, Boston, Mass, USA, 2003.

[3] M. Adivar and Y. N. Raffoul, "Existence results for periodic solutions of integro-dynamic equations on time scales," Annali di Matematica Pura ed Applicata, vol. 188, no. 4, pp. 543-559, 2009.

[4] F. M. Atici, D. C. Biles, and A. Lebedinsky, "An application of time scales to economics," Mathematical and Computer Modelling, vol. 43, no. 7-8, pp. 718-726, 2006.

[5] H. D. Liu and F. W. Meng, "Some new generalized VolterraFredholm type discrete fractional sum inequalities and their applications," Journal of Inequalities and Applications, vol. 2016, article 213, p. 16, 2016.

[6] A. Dogan, "On the existence of positive solutions of the pLaplacian dynamic equations on time scales," Mathematical Methods in the Applied Sciences, vol. 40, no. 12, pp. 4385-4399, 2017.

[7] A. Dogan, "Positive solutions of the p-Laplacian dynamic equations on time scales with sign changing nonlinearity," Electronic Journal of Differential Equations, vol. 39, pp. 1-17, 2018.

[8] H. D. Liu and F. W. Meng, "Existence of positive periodic solutions for a predator-prey system of Holling type IV function response with mutual interference and impulsive effects," Discrete Dynamics in Nature and Society, vol. 2015, Article ID 138984, 12 pages, 2015. 
[9] D. L. Zhao and H. D. Liu, "Coexistence in a two species chemostat model with Markov switchings," Applied Mathematics Letters, vol. 94, pp. 266-271, 2019.

[10] L. Erbe, B. G. Jia, and A. Peterson, "On the asymptotic behavior of solutions of Emden-Fowler equations on time scales," Annali di Matematica Pura ed Applicata, vol. 191, no. 2, pp. 205-217, 2012.

[11] H. D. Liu and F. W. Meng, "Interval oscillation criteria for second-order nonlinear forced differential equations involving variable exponent," Advances in Difference Equations, no. 291, p. 14, 2016.

[12] M. Federson, J. G. Mesquita, and A. Slavik, "Measure functional differential equations and functional dynamic equations on time scales," Journal of Differential Equations, vol. 252, no. 6, pp. 3816-3847, 2012.

[13] E. Tunç and H. D. Liu, "Oscillatory behavior for second-order damped differential equation with nonlinearities including Riemann-Stieltjes integrals," Electronic Journal of Differential Equations, vol. 2018, article 54, p. 12, 2018.

[14] H. D. Liu, "Some new integral inequalities with mixed nonlinearities for discontinuous functions," Advances in Difference Equations, no. 22, p. 16, 2018.

[15] L. Erbe, B. Jia, and A. Peterson, "Belohorec-type oscillation theorem for second order sublinear dynamic equations on time scales," Mathematische Nachrichten, vol. 284, no. 13, pp. 16581668, 2011.

[16] D. Hu and Z. Zhang, "Four positive periodic solutions to a Lotka-Volterra cooperative system with harvesting terms," Nonlinear Analysis: Real World Applications, vol. 11, no. 2, pp. 1115-1121, 2010.

[17] H. D. Liu and F. W. Meng, "Some new nonlinear integral inequalities with weakly singular kernel and their applications to FDEs," Journal of Inequalities and Applications, no. 209, p. 17, 2015.

[18] A. Slavik, "Averaging dynamic equations on time scales," Journal of Mathematical Analysis and Applications, vol. 388, no. 2, pp. 996-1012, 2012.

[19] H. D. Liu, "Lyapunov-type inequalities for certain higher-order difference equations with mixed non-linearities," Advances in Difference Equations, no. 229, p. 14, 2018.

[20] Z. Q. Zhang and J. D. Cao, "Periodic solutions for complexvalued neural networks of neutral type by combining graph theory with coincidence degree theory," Advances in Difference Equations, vol. 2018, no. 261, p. 23, 2018.

[21] B. Karpuz, "Volterra theory on time scales," Results in Mathematics, vol. 65, no. 3-4, pp. 263-292, 2014.

[22] S. Hilger, Ein Maßkettenkalkül mit Anwendung auf Zentrumsmanningfaltigkeiten [Ph.D. thesis], Universität Würzburg, 1988.

[23] R. Agarwal, M. Bohner, and A. Peterson, "Inequalities on time scales: a survey," Mathematical Inequalities \& Applications, vol. 4, no. 4, pp. 535-557, 2001.

[24] D. R. Anderson, "Nonlinear dynamic integral inequalities in two independent variables on time scale pairs," Advances in Dynamical Systems and Applications (ADSA), vol. 3, no. 1, pp. 1-13, 2008.

[25] H. D. Liu, "An improvement of the Lyapunov inequality for certain higher order differential equations," Journal of Inequalities and Applications, no. 215, p. 9, 2018.

[26] L. Du and R. Xu, "Some new Pachpatte type inequalities on time scales and their applications," Journal of Mathematical Inequalities, vol. 6, no. 2, pp. 229-240, 2012.
[27] F. Meng and J. Shao, "Some new Volterra-Fredholm type dynamic integral inequalities on time scales," Applied Mathematics and Computation, vol. 223, pp. 444-451, 2013.

[28] W. N. Li and W. Sheng, "Some nonlinear integral inequalities on time scales," Journal of Inequalities and Applications, Article ID 70465, p. 15, 2007.

[29] W. N. Li and M. Han, "Bounds for certain nonlinear dynamic inequalities on time scales," Discrete Dynamics in Nature and Society, vol. 2009, Article ID 897087, 14 pages, 2009.

[30] E. A. Bohner, M. Bohner, and F. Akin, "Pachpatte inequalities on time scale," Journal of Inequalities in Pure and Applied Mathematics, vol. 6, no. 1, article no. 6, 2005.

[31] S. H. Saker, "Some nonlinear dynamic inequalities on time scales," Mathematical Inequalities \& Applications, vol. 14, no. 3, pp. 633-645, 2011.

[32] Y. Sun and T. Hassan, "Some nonlinear dynamic integral inequalities on time scales," Applied Mathematics and Computation, vol. 220, pp. 221-225, 2013.

[33] Q. Feng, F. Meng, and B. Zheng, "Gronwall-Bellman type nonlinear delay integral inequalities on time scales," Journal of Mathematical Analysis and Applications, vol. 382, no. 2, pp. 772$784,2011$.

[34] H. D. Liu, "On some nonlinear retarded Volterra-Fredholm type integral inequalities on time scales and their applications," Journal of Inequalities and Applications, no. 211, p. 19, 2018.

[35] D. L. Zhao, S. L. Yuan, and H. D. Liu, "Random periodic solution for a stochastic SIS epidemic model with constant population size," Advances in Difference Equations, no. 64, p. 9, 2018.

[36] H. D. Liu, "A class of retarded Volterra-Fredholm type integral inequalities on time scales and their applications," Journal of Inequalities and Applications, no. 293, p. 15, 2017.

[37] Y.Z. Tian, Y. L. Cai, L. Z. Li, and T. X. Li, "Some dynamic integral inequalities with mixed nonlinearities on time scales," Journal of Inequalities and Applications, vol. 2015, article 12, pp. 1-10, 2015.

[38] H. D. Liu and F. W. Meng, "Nonlinear retarded integral inequalities on time scales and their applications," Journal of Mathematical Inequalities, vol. 12, no. 1, pp. 219-234, 2018.

[39] J. Wang, F. Meng, and J. Gu, "Estimates on some power nonlinear Volterra-Fredholm type dynamic integral inequalities on time scales," Advances in Difference Equations, vol. 2017, article 257, p. 16, 2017.

[40] J. Gu and F. W. Meng, "Some new nonlinear Volterra-Fredholm type dynamic integral inequalities on time scales," Applied Mathematics and Computation, vol. 245, pp. 235-242, 2014.

[41] W. N. Li and W. Sheng, "Some Gronwall type inequalities on time scales," Journal of Mathematical Inequalities, vol. 4, no. 1, pp. 67-76, 2010.

[42] F. C. Jiang and F. W. Meng, "Explicit bounds on some new nonlinear integral inequalities with delay," Journal of Computational and Applied Mathematics, vol. 205, no. 1, pp. 479-486, 2007. 


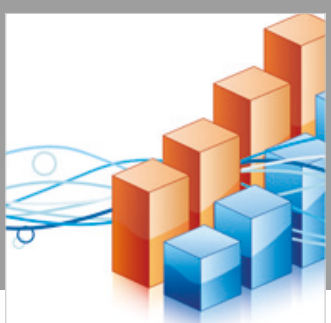

Advances in

Operations Research

\section{-n-m}
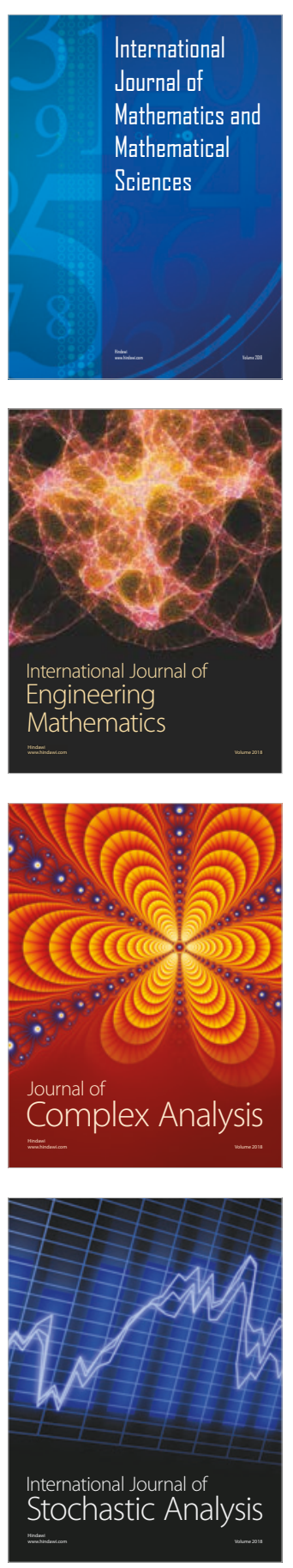
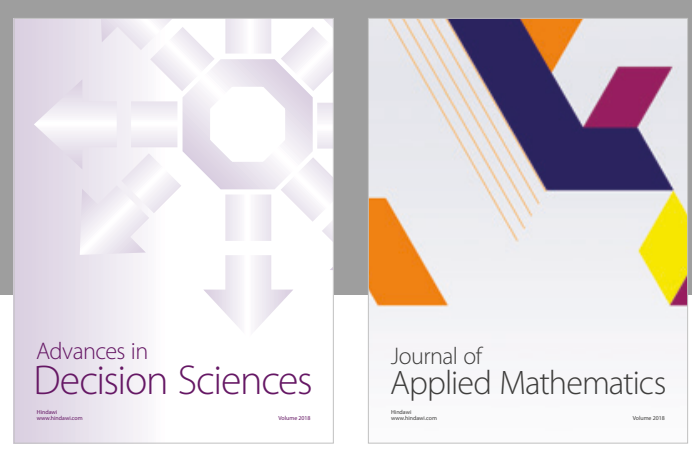

Journal of

Applied Mathematics
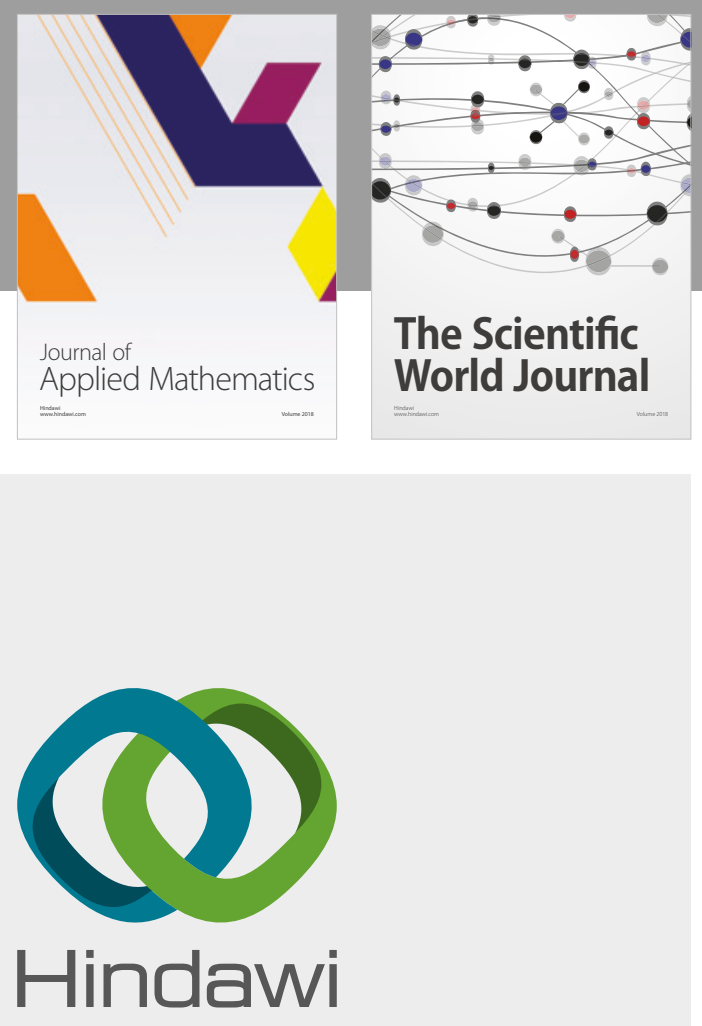

Submit your manuscripts at

www.hindawi.com

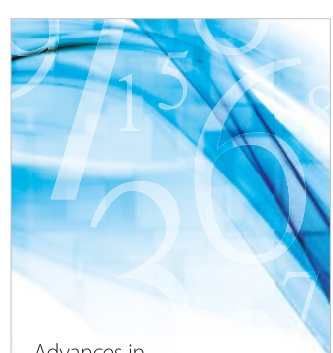

Advances in
Numerical Analysis
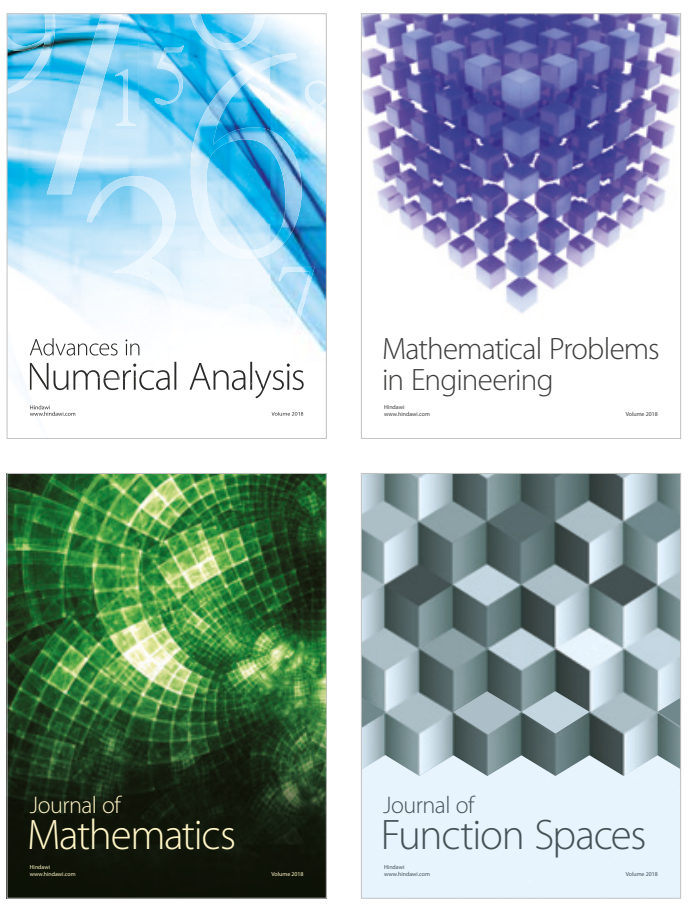

Mathematical Problems in Engineering

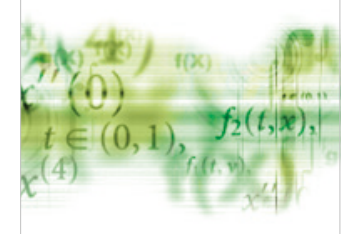

International Journal of

Differential Equations

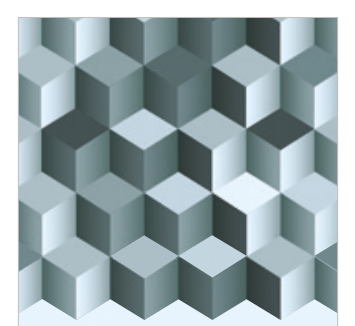

Journal of

Function Spaces

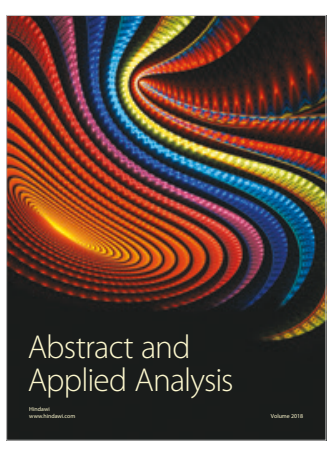

The Scientific

World Journal

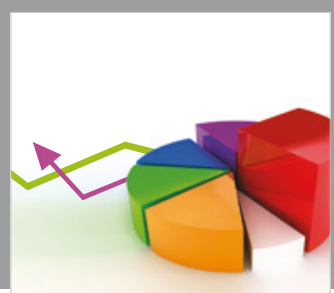

Journal of

Probability and Statistics
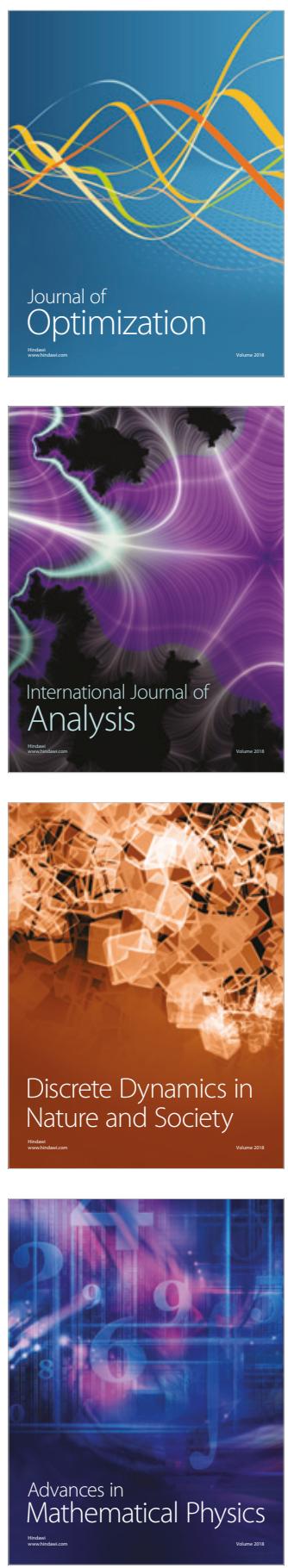